\title{
Influence of negative Poisson's ratio on stent applications
}

\author{
S. K. Bhullar ${ }^{1, ~ *, ~ A . ~ T . ~ M a w a n a n e ~ H e w a g e ~}{ }^{2}$, A. Alderson ${ }^{2}$, K. Alderson ${ }^{2}$, Martin B. G. Jun ${ }^{1}$ \\ ${ }^{1}$ Dept. of Mechanical Engineering, University of Victoria, Victoria, British Columbia,Canada \\ ${ }^{2}$ Institute for Materials Research \& Innovation, University of Bolton, Deane Road, Bolton, U.K.
}

\section{Email address:}

sbhullar@uvic.ca(S. K. Bhullar)

\section{To cite this article:}

S. K. Bhullar, A. T. MawananeHewage, A. Alderson, K. Alderson, Martin B. G. Jun. Influence of Negative Poisson's Ratio on Stent Applications. Advances in Materials. Vol. 2, No. 3, 2013, pp. 42-47. doi: 10.11648/j.am.20130203.14

\begin{abstract}
Stents are usually defined as small tubular structures that are inserted into the diseased region which provide mechanical support of damaged artery or some other hollow organ to restore lumen and blood flow conditions in arteries. Recently developed stents may accumulate functions; for example, they can be used for drug delivery or for treatment of bleeding esophageal varices. Further clinical failures such as crushing, removing, restenosis are some of the most serious reasons for continuing stent improvements. Therefore, our research study has the design and manufacture of an auxetic structure with enhanced mechanical properties through the tailored negative Poisson's ratio, and its configuration as an auxetic stent to be used for example in the palliative treatment of esophageal cancer and for the prevention of dysphagia as the main objective. Background: The use of stents for esophageal diseases has evolved greatly over the past 30 years. Esophageal cancer is the growth of cancer cells in the esophagus tube which usually originates in the inner layers of the lining of the esophagus and grows outward. In time, the tumor can obstruct the passage of food and liquid, making swallowing painful and difficult. Since most patients are not diagnosed until the late stages of the disease, esophageal cancer is associated with poor quality of life and low survival rates. Method: This research study is organized in three parts devoted to fabrication of auxetic structure and stent samples, experimental testing and discussion of results. For this purpose a model of an auxetic rotating-square geometry with a circular hole structure is developed by laser cutting apolyurethane sheet. The auxeticoesophageal stent was made by folding and gluing the auxetic sheet (with rigid squares and circular holes) into a cylindrical shape. Combined videoextensometry with compressive and tensile testing are employed to determine the deformation response of the auxetic sheet and stent to obtain stress-strain curves and estimation of Poisson's ratio.Conclusions: The stenting outcomes are improved through mechanical design with tailored negative Poisson's ratio through cell geometry of the esophagus stent. Theauxetic stent geometry of rotating squares with circular holes will be lighter in weight; reduces obstruction and food impaction because of its unique deformation mechanism; and can reduces migration of the implant stent by embedding inside the tissue.
\end{abstract}

Keywords: Negative Poisson's Ratio, AuxeticStents, Synclastic Property of Materials, Rotating Squares Geometry with Holes

\section{Introduction}

The esophagus is the tube that runs from the mouth to the stomach and it carries food we swallow to our stomach to be digested. Esophageal cancer is the growth of cancer cells in this tube and cancer occurs when cells in the body divide out of control. If cells keep dividing, a mass of tissue forms and these are called growths or tumors. If the tumor is malignant, it is cancer and it can invade nearby tissue and spread to other parts of the body [1]. Esophageal tumors usually lead to dysphagia (difficulty swallowing), pain and other symptoms [2] and usually originate in the inner layers of the lining of the esophagus and grow outwards. There isgreat variability in the incidence of esophageal cancer with regard to geography, ethnicity, and gender. The overall incidence is increasing [3] and about 13,000 new cases of esophageal cancer are diagnosed in the United States each year. During the same 12-month period, 12,000 people die of this disease. It strikes between five and ten North Americans per 100,000. In some areas of China the cancer is endemic. In general, esophageal cancer occurs more frequently in men than in women. Treatment for esophageal cancer is determined by the stage of the disease and the patient's general health. The most important distinction to make is whether the cancer is curable. If the cancer is in the early stages, cure may be possible. If the cancer is advanced than in addition to surgery, 
chemotherapy, and radiation, palliative measures can provide symptomatic relief. Stents are used as a safe and effective alternative surgery to improve quality of life for patients. For example dilatation of the narrowed portion of the esophagus with soft tubes can provide short-term relief of dysphagia and placement of a flexible stent [4] as illustrated in Figure 1, is useful in allowing more food intake. The following section gives a brief overview of stent designs.

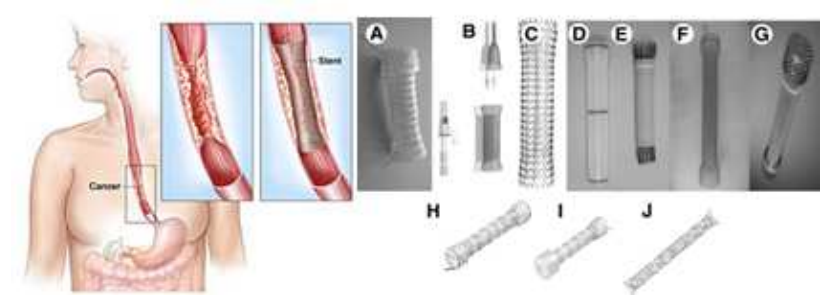

Figure 1. Esophageal cancer affecting the lower esophagusboth before and after placement of a stent(left) and some examples of stents(right) [4].

\subsection{Stent Design Overview}

Hollow tubes or stents placed inside malignant strictures can maintain a patent lumen and alleviate dysphagia. The insertion of tubes made of plastic materials has been successful,but associated with a $\leq 15 \%$ risk of acute complications during insertion, particularly esophageal perforation [5-6]. Recently developed expandable metal stents as shown in Figure 1(right) [7] are more expensive, but they are much easier to insert with a significantly lower rate of acute complications. Current stent technology typically utilizes a wire-guided delivery device to pass a compressed and constrained mesh stent across the area to be treated. Metal stents have become popular for the palliation of patients with malignant esophageal obstruction, especially patients with a poor prognosis [8-11]. At present more than 100 types of stent are available on the market [12], classified in accordance with their clinical use (vascular or nonvascular, coronary or peripheral). From the more technical point of view the stents may be differentiated by material, form of material and design. The two main groups are balloon-expandable and self-expandingstents[13-14]. The balloon-expandable stents are manufactured with a smaller diameter and, after delivery, they are dilated with a balloon. Thus the material used must be able to be plastically deformed and to remain in the expanded shape. It means it should have a low yield stress. On the other hand, self-expanding stents are manufactured so that their rest diameter is greater than the inner diameter of the target structure. The material used should have in the ideal case a low elastic modulus and a high yield stress [15-16]. Also, it has been found that enhanced mechanical properties of smart materials tailored through a negative Poisson's ratio, called auxetic materials, are beneficial in stent design. These materials have high shear modulus, for example when Poisson's ratio approaches $\mathbf{- 1}$, the shear modulus substantially exceeds the Young's modulus. A small Young's modulus combined with large toughness, stiffness without brittleness, synclasticbehaviour and resilient nature make auxetic materials good candidates for the biomedical industry. The next section describes a brief introduction of auxetic materials.

\subsection{AuxeticMaterials}

Many materials (a rubber band, for example) attempt to maintain their volume when stretched by becoming thinner. However the materials with negative Poisson's ratio called auxetic materials become wider when stretched[17]. Auxetic structures can have enhanced and improved properties over conventional materials such as increased indentation resistance, good absorption properties (acoustic absorption), and higher fracture toughness [18-19]. Scientists have been aware of the existence of auxetic materials for over a hundred years.For example, Love [20] presented an example of a cubic single crystal pyrite as having a Poisson's ratio of -0.14 . Some very important biological materials are auxetic too. The first material to be considered was skin and it has been found that several types of skin possess a negative Poisson's ratio such as cat skin [21], cow teat skin [22] and salamander skin [23]. A further natural auxetic material that has been studied and predicted to have a negative Poisson's ratio is the load-bearing cancellous bone from human shins [24]. Another example of a tissue that demonstrates a negative Poisson's ratio is the arterial endothelium when subjected to both wall shear stresses and a cyclic circumferential strain due to pulsatile blood flow [25]. A scaffold made out of an auxetic material (an auxetic scaffold) would expand and contract in tandem with the strains resulting from the cyclical pressures from pulsatile blood flow. Thus, an auxetic scaffold that exhibits concurrent axial and transverse expansion (contraction) would likely better integrate with native tissues and better promote clinical tissue regeneration. Other examples where auxetic constructs could be useful are in myocardial tissue engineering (as an auxetic cardiac patch), skin and fat tissue engineering and in wound management (e.g., auxetic medical sutures) [26-29]. Further, the increasing trend to incorporate artificial devices such as bone and joint replacements, dental prostheses, implanted sensors and heart valves into the human body, has sharply focused attention on to the compatibility of the materials from which such devices are made with human physiology and chemistry. Therefore, it is a major current challenge in biomaterials to develop implants that are truly permanent and, to design and manufacture such materials to make them maximally compatible with living tissue. Studies and experiments demonstrate that polyurethanes with tailored negative Poisson's ratio offer a huge potential in biomedical applications such as the fabrication of stents, scaffolds, implants and prostheses.

With the above background the design and manufacture of an auxetic structure and its configuration into an auxetic stent to be used, for example, in the palliative treatment of esophagealcancer, and for the prevention of dysphagia is targeted in this study. For this purpose a model of an auxeticrotating-square geometry with a circular hole 
structure is developed by laser cutting of apolyurethane sheet. The motivation of this work is inspired by an auxetic structure configured as an esophageal stent with potential to be used for the palliative treatment of esophageal cancer [30] employing anauxetic (rotating squares) geometry theoretically predicted in [31]. Therefore, the main focus in our research study is to acquire the mechanical properties of the auxetic rotating solid squares geometry with circular holes and without holes (solid squares) based on an arrangement involving rigid squares connected together at their vertices by hinges. Each unit cell contains four squares, each square contains four vertices, and two vertices correspond to one hinge. The following is the methodology and modeling technique, and the discussion of the results.

\section{Method and Solution}

\subsection{Design of the AuxeticStructure}

The arrangement involves rigid squares with circular holes (i.e., rotating-square geometry) connected together at their vertices through hinges by using computerized numerical control (CNC) guided laser cutting machine. A rotating-square geometry as illustrated in Figure 2(left), was designed using the SolidWorks engineering software. Polyurethane sheets $(210 \mathrm{~mm} \times 297 \mathrm{~mm} \times 2 \mathrm{~mm})$ were used to prepare samples using the laser cutter (World lasers LR1612) see Figure 2 (right). 5\% power of the $40 \mathrm{~W} \mathrm{CO}_{2}$ laser beam with the beam speed of $10 \mathrm{mms}^{-1}$ was used in this work. The corner power used in this work is $2 \%$ of the main beam.

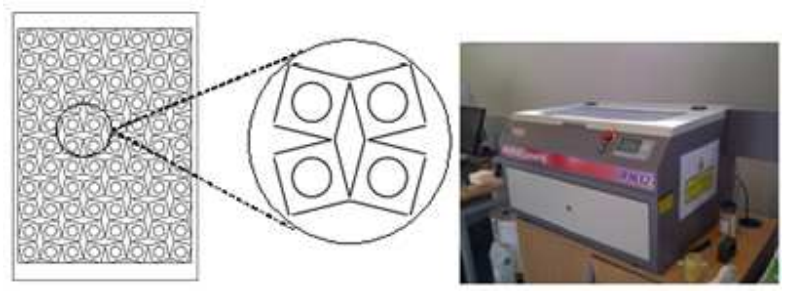

Figure 2. A rotating-square geometry with holes (left), World lasers LR1612 (right)

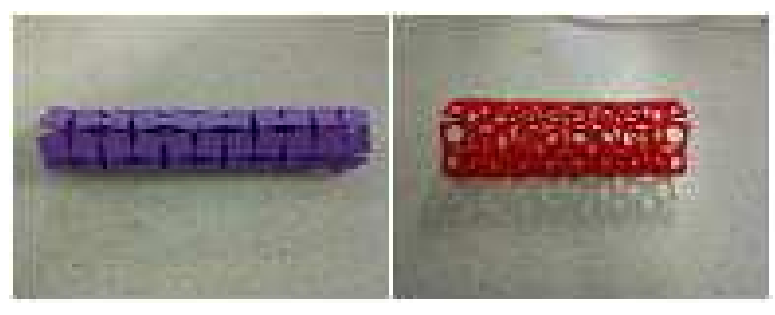

Figure 3. Cylindrical shaped stents fabricated from auxetic sheets with rotating solid squares (left) and rotating solid squares with holes (right).

The Auxeticesophageal polyurethane stent was made by folding the polyurethane sheet (with rigid squares and circular holes) into a cylindrical shape. UHU super glue was used to adjoin the edges of the sheets to make the cylinder as shown in Figure 3. The dimensions of the stents were $234 \mathrm{~mm}$ long, internal diameter $38 \mathrm{~mm}$, outer diameter 42 $\mathrm{mm}$ (Figure 3 left), and 170mm long, internal diameter49 $\mathrm{mm}$ and outer diameter $53 \mathrm{~mm}$ (Figure 3 right).

\subsection{Mechanical Properties Characterisation}

\subsubsection{Tensile Tests}

AnInstron 3369 mechanical testing machine, at a cross head speed of $2 \mathrm{~mm} / \mathrm{min}$ up to $2 \mathrm{~mm}$ extensionwas used for the tensile tests as shown in Figure 4.Mechanical properties characterisationcomprised axial and transverse strains for Poisson' ratio estimation under uniaxial tension. The percentage longitudinal strain with corresponding value of applied load was measured to analyse the elastic and plastic deformation behaviour under uniaxial tensile load of the auxetic sheets of $205(\times) 185(\times) 2 \mathrm{~mm}$ and $250(\times) 180(\times) 2 \mathrm{~mm}$ sheets with end tabs. The load-displacement data were recorded on the InstronPC for sheets comprising rotating solid square and rotating squares with circular holes, and corresponding values of engineering stress and strain were calculated by using equation (1). The depicted stress-strain curve is shown in Figure 5.

$$
\sigma=\frac{l o a d}{\operatorname{area}} \text { and } \varepsilon=\frac{\Delta L}{L_{0}}
$$

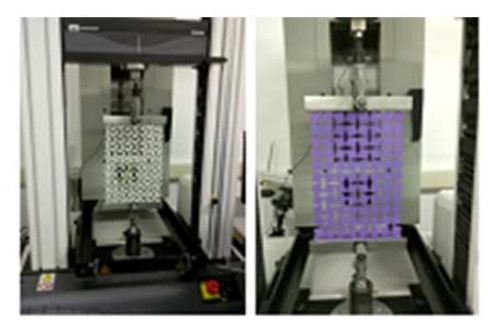

Figure 4. Instron 3369 mechanical testing machine and auxetic sheets with end tabs for tensile tests.

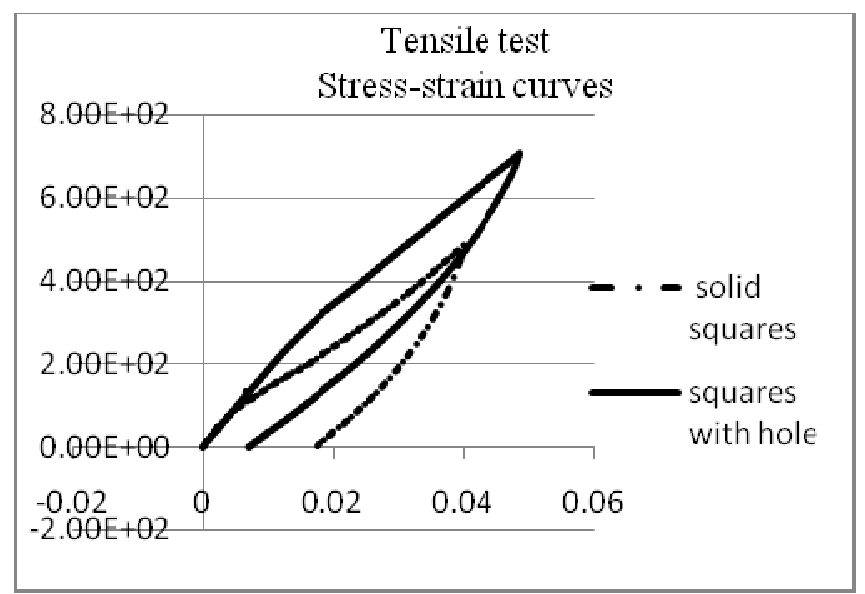

Figure 5. Comparison of stress-strain curves for rotating solid squares and rotating squares with circular holes

The hysteresis present in the stress - strain graphs in Figure 5 shows that upon removal of the tensile load the samplesdo not return to their original positions (origin), which indicates plastic deformation. It is noticed that the plastic deformation is less in theauxetic structure with holes compared to the auxetic structure without holes in the squares. 


\subsubsection{The Compressive Stress-Strain Behaviour}

Combined videoextensometry and compression testing wasemployed to determine the axial compression response of the auxetic stent. The auxetic stent samples with the geometry of solid squares and squares with circular holes were subjected to an axial compression using an Instron 3369 testing machine as illustrated in Figure 6. The stents were placed between the parallel compression plates of the machine and compressed at a constant cross-head speed of 2 $\mathrm{mm} / \mathrm{min}$ whilst simultaneously undertaking axial and transverse compression measurements from the movement of fiducial markers placed on the auxetic stents using a MESSPHYSIK ME 46 videoextensometer. Each stent sample was tested 3 times and the average value of Poisson's ratio $v$ from the videoextensometrydata was estimated. The compressive stress-strain behaviour of the auxetic stents for all three tests on each stentare shown in Figure 7 and Figure 8.
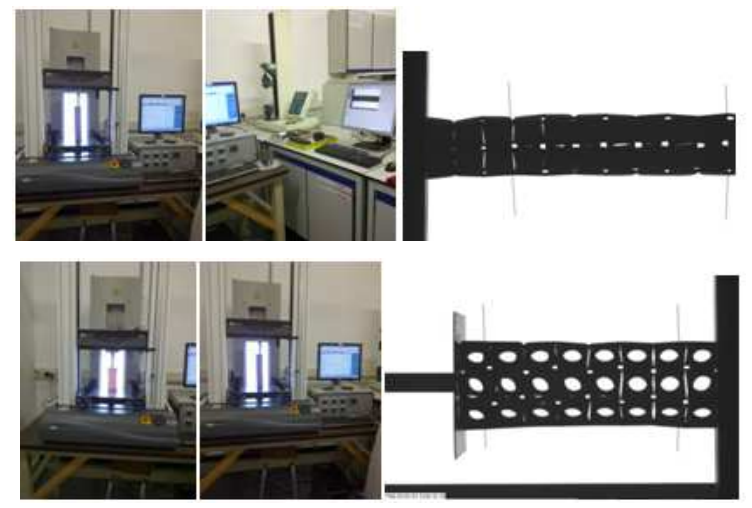

Figure 6. The compressive tests of the auxetic stent samples using instron and videoextensometry - rotating solid squares (top) and squares with circular holes (bottom)

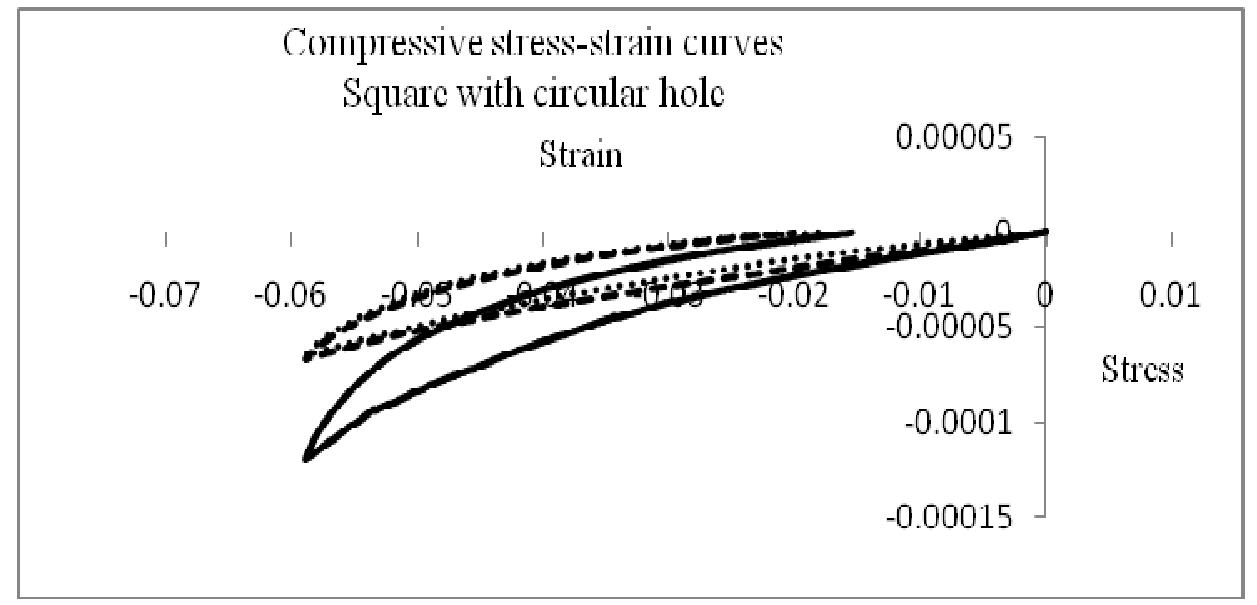

Figure 7. Compressive stress-strain curves for auxetic stent ofrotating squares with circular holes.

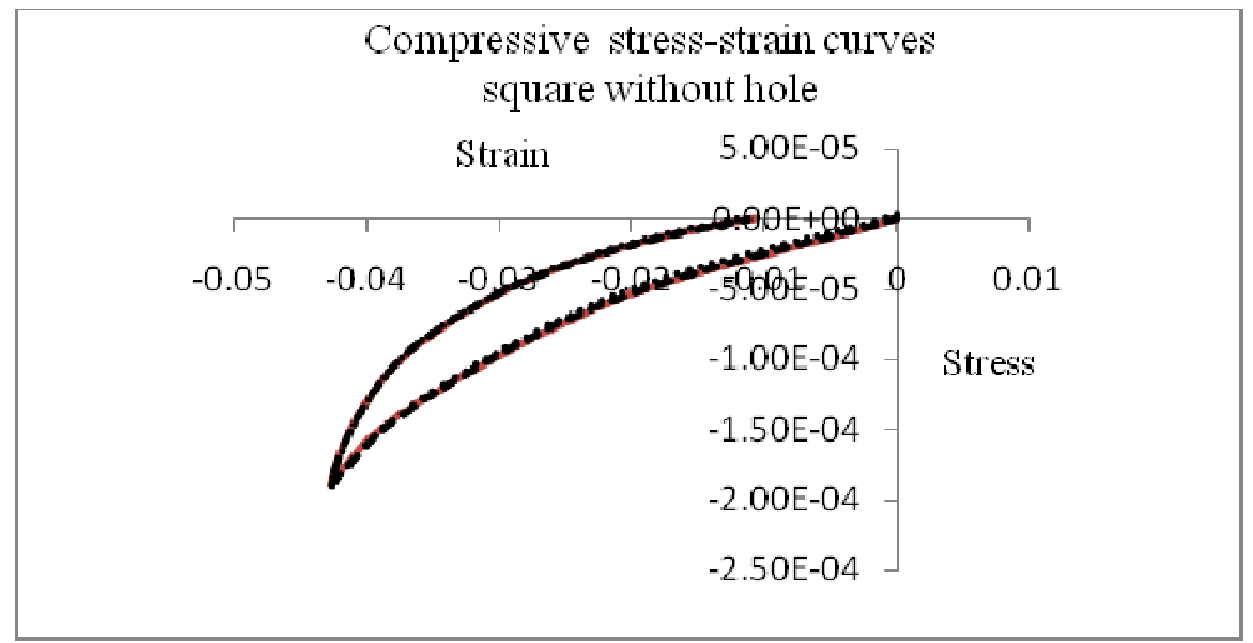

Figure 8. Compressive stress-strain curves for auxetic stent of rotating squares without circular holes.

The stress - strain curves of both auxetic stents due to compressive testingshow hysteresis during loading and relaxing. In all the three tests the curves do not return to the original position, showing plastic deformation of the auxetic stents. The Poisson's ratio of the stent samples was calculated from the videoextensometry data using equation (2):

$$
v=-\frac{\varepsilon_{y}}{\varepsilon_{x}}
$$


where $\varepsilon_{\mathrm{y}}$ and $\varepsilon_{\mathrm{x}}$ are the transverse and axial strains, respecti vely.

The maximum Poisson's ratio calculated for stent with circular holes and without circular holes is shown in Table 1.The average Poisson's ratio is calculated as $v=-0.76$ for auxetic stent with holes and $v=-0.72$ in the case of auxetic stent without holes.

\section{Conclusions}

In this paper we have reported fabrication and experimental studies of auxetic esophageal stents of rotating solid squares and rotating squarea with circular holes having enhanced mechanical properties through the tailored negative Poisson's ratio. The stents with the geometry of rotating squares with holes having average Poisson's ratio of $v=-0.76$ are lighter in weight compared to auxetic stents without holes having Poissons's ratio of $v=-0.72$

\subsection{Study Highlights}

\subsubsection{What Is Current Knowledge}

Stents are often used as a safe and effective alternative to surgery or repetitive endoscopic procedures to improve quality of life for patients with esophageal or gastric cardia cancer. The recent developments of self-expanding plastic stents and self-expanding metal stents, and stent placement for esophageal pathology can be safe and cost effective. However, the potential complications of esophageal stenting include stent migration, obstruction from tumour ingrowth or overgrowth and food impaction. There is a need to improve designs of stents that should overcome the problems encountered with the existing stents.

\subsubsection{What Is New In Our Research Study}

The stenting outcomes are improved through mechanical design with tailored negative Poisson's ratio through cell geometry of the esophagus stent. The property of getting wider when stretched for anauxetic stent along with enhanced mechanical properties such as synclasticbehaviour and stiffness without brittleness can help minimize the stresses. The auxetic stent comprising rotating squares with circular holes is lighter in weight. It can be helpful to reduce the obstruction and food impaction because of its large (expandable) diameter due to the auxeticdeformation mechanism. Also, it can achieve improved anchorage with the surrounding tumour tissue inside the esophagus. to the auxetic stent can achieve a good grip with tumour tissue by embedding inside the tissue and can reduce migration of the implant stent.Further developments will include collaboration with researchers with interests in biological devices together with local health authorities, design and manufacturing companies relating to the compatiability of the auxetic stent with circular holes.
Table 1. The Maximum Poisson's ratio calculated for Stent with and without holes

\begin{tabular}{|c|c|c|}
\hline Experimental Data & $\begin{array}{l}\text { Stent with hole } \\
\text { Poisson's ratio }\end{array}$ & $\begin{array}{l}\text { Stent without hole } \\
\text { Poisson's ratio }\end{array}$ \\
\hline $\begin{array}{c}\text { Test 01(gradient } \\
\text { testing) }\end{array}$ & -0.6 & -0.58 \\
\hline Test 02 & -0.98 & -0.57 \\
\hline Test 03 & -0.98 & -0.59 \\
\hline $\begin{array}{l}\text { Test 01(based } \\
\text { equation 2) }\end{array}$ & -0.71 & -0.60 \\
\hline Test 02 & -0.66 & -0.56 \\
\hline Test 03 & -0.73 & -0.64 \\
\hline $\begin{array}{c}\text { Test01 } \\
\text { (Videoextensometry) }\end{array}$ & -0.34 & -0.83 \\
\hline Test 02 & -0.76 & -0.65 \\
\hline Test 03 & -1.03 & -1.54 \\
\hline Average & -0.76 & -0.72 \\
\hline
\end{tabular}

\section{Acknowledgements}

We are really thankful to Mohammad Sanami of the Institute for Materials Research \& Innovation, University of Bolton, Deane Road, Bolton, U.K. for assistance with the experimental testing

\section{References}

[1] Enzinger PC, Mayer RJ, "Esophageal cancer". N. Engl. J. Med. 2003,349 (23): 2241-52, doi:10.1056/NEJMra035010. PMID 14657432.

[2] Shield W, Thomas. LoCicero, Joseph. B. Ponn, Ronald. Less Common Malignant Tumors of the Esophagus. Lippincott Williams \& Wilkins 2005, pp. 2325-2340. ISBN 978-0-7817-3889-7.

[3] Boyce HW Jr, Palliation of advanced esophageal cancer. SeminOncol 1984;11:186 .95.

[4] Ahmed ME, Gustavson S. Current palliative modalities for esophageal car-cinoma. ActaChirScand 1990;156:95.8.

[5] Tytgat GNJ. Endoscopic therapy of esophageal cancer: Possibilities and limitations. Endoscopy 1990;22:263.7.

[6] Knyrim K, Wagner HJ, Bethge N, et al. A controlled trial of an expansile stent for palliation of esophageal obstruction due to inoperable cancer. N Engl J Med 1993;329:1302.7.

[7] Homs MY, Steyerberg EW, Eijkenboom WM, et al. Single-dose brachytherapy versus metal stent placement for the palliation of dysphagia from oesophageal cancer: multicentrerandomised trial. Lancet 2004;364:1497-504. 
[8] Steyerberg EW, Homs MY, Stokvis A, et al. Stent placement or brachytherapy for palliation of dysphagia from esophageal cancer: a prognostic model to guide treatment selection. GastrointestEndosc 2005;62: 333-40.

[9] Homs MY, Kuipers EJ, Siersema PD. Palliative therapy. J SurgOncol 2005;92:246-56.

[10] Siersema PD. Treatment options for esophageal strictures. Nat ClinPractGastroenterolHepatol 2008;5:142-52.9

[11] Costamagna G, Marchese M, Iacopini F. Self-expanding stents in oesophageal cancer. Eur J GastroenterolHepatol 2006;18:1177-80.

[12] Mougey A, Adler DG. Esophageal stenting for the palliation of malignant dysphagia. J Support Oncol 2008;6:267-73.

[13] Siersema PD, Marcon N, Vakil N. Metal stents for tumors of the distal esophagus and gastric cardia. Endoscopy 2003;35:79-85

[14] Saranovic D, Djuric-Stefanovic A, Ivanovic A, et al. Fluoroscopically guided insertion of self-expandable metal esophageal stents for palliative treatment of patients with malignant stenosis of esophagus and cardia: comparison of uncovered and covered stent types. Dis Esophagus $2005 ; 18: 230-8$

[15] Conio M, Repici A, Battaglia G, et al. A randomized prospective comparison of self-expandable plastic stents and partially covered self-expandable metal stents in the palliation of malignant esophageal dysphagia. Am J Gastroenterol 2007;102:2667-77.

[16] Stavroulakis GE. Auxeticbehaviour: appearance and engineering applications. Phys Status Solidi. $2005 ; 242(3): 710.20$

[17] Lakes, RS,Foam structures with a negative Poisson's ratio. Science, 1987a,235: 1038-1040.

[18] Lakes,RS.,Polyhedron cell structure and method of making same. Int. Patent.,1987b Publ. No. WO88/00523.

[19] Lakes, RS.,Design considerations for negative Poisson's ratio materials. ASME J. Mech. Design, 1993a.115,.696-700.
[20] Love, A.E. H., A Treatise on the Mathematical Theory of Elasticity, Dover, New York, NY, USA, 4th edition, Cambridge University Press,Cambridge,1927,163.

[21] Veronda, DR.Westmann, RA. Mechanical characterization of skin finite deformations.,J.Biomech., 1970, 3, 111.124.

[22] Frohlich, LM. LabarberaStevens,WP. Poisson.s ratio of a crossed fibre sheath: the skin of aquatic salamanders, J. Zool. Lond.,1994,232, 231.252.

[23] Lees, C., Vincent, JEV.,Hillerton, JE., .Poisson.s ratio in skin., Biomed.Mater. Eng., 1991,1, 19.23.

[24] Williams, JL. and Lewis, JL.Properties and an anisotropic model of cancellous bone from the proximal tibialepiphysis.Trans.ASME,J.Biomech. Eng.,1982,104, 5.56.

[25] Liulan, L., Qingxi, H, Xianxu, H., Gaochun, X. .Design and Fabrication of Bone Tissue Engineering Scaxoolds via Rapid Prototyping and CAD., Journal of Rare Earths,2007, 25(2), 379.383 .

[26] Pan, Y., Dong, S., Hao, Y., Chu,T., Li, C., Zhang Z., Zhou,Y.,.Demineralized bone matrix gelatin as scaffold for tissue engineering., African Journal of Microbiology Research 2010, 4(9), 865-870.

[27] Yang W, et al. Review on auxetic materials. J Mater Sci.2004;39:3269.79.

[28] Gaspar N, et al. Quantitative analysis of the microscale of auxetic foams. Phys Status Solidi. 2005;242(2):550.60.

[29] Alderson A, et al. Auxetic polymeric filters display enhanced defouling and pressure compensation properties. Membrane Technol. 2001;137:6.8.

[30] Ali MN.Rehman IU. An Auxetic structure configured as oesophageal stent with potential to be used for palliative treatment of oesophageal cancer; development and in vitro mechanical analysis, J Mater Sci: Mater Med 2011, 22: DOI 10.1007/s10856-011-4436-y, pp. 2573-2581.

[31] Grima JN, et al. Do zeolites have negative Poisson.s ratios? Adv Mater. 2000;12(24):1912.8. 